\title{
Single emitter fluorescence enhancement with surface lattice resonances
}

\author{
Frédéric Laux, ${ }^{\dagger}$ Nicolas Bonod, ${ }^{\ddagger}$ and Davy Gérard*,† \\ $\dagger$ Laboratoire de Nanotechnologie et Instrumentation Optique, Institut Charles Delaunay, \\ UMR CNRS 6281, Université de Technologie de Troyes, France \\ $\ddagger$ Aix-Marseille Univ, CNRS, Centrale Marseille, Institut Fresnel, Marseille, France \\ E-mail: davy.gerard@utt.fr
}

\begin{abstract}
Surface lattice resonances (SLRs) are high quality factor resonances arising in periodic arrays of nanoantennas that offer unique opportunities to enhance light-matter interactions. SLRs have been successfully used to enhance non-linear signals, magnetooptical effects and also to enhance the fluorescence signal from quantum emitters. However, the high potential of SLRs to tailor light emission of single photon sources is still unexplored. In this study, we report numerical investigations of light emission from a single emitter coupled to SLRs. We demonstrate that the excitation of SLRs with an impinging plane wave allows for the enhancement of the local field intensity by several orders of magnitude, much higher than what can be achieved with localized resonances. We also study the alteration of the decay rate of the emitter (Purcell effect) inside the array, evidencing an emission enhancement similar to the one of the localized resonance of a single antenna. Finally, we plot the far-field radiation patterns of the arrays and show that they are controlled by the period of the arrays and the emission wavelength, enabling photon sorting with the emission angle. Altogether, our study demonstrates a new route to design high-brightness sources based on single emitters.
\end{abstract}




\section{Introduction}

Optical antennas are nano-sized devices transducing optical energy from the near-field (localized energy) to the far-field (propagating waves), and vice-versa. ${ }^{1}$ This unique ability to control light at the nanoscale has been massively used to engineer the radiative properties of emitters. ${ }^{2-4}$ Nanoantennas are often based on metallic nanostructures sustaining localized surface plasmon resonances (LSPRs), optical modes that are confined within a few nanometers of the metal surface. ${ }^{5}$ Light emitters, such as quantum dots or fluorescent molecules, coupled to nanoantennas have been reported to exhibit directional emission, ${ }^{6}$ high brightness, ${ }^{7,8}$ ultrafast emission ${ }^{9}$ and high quantum yield. ${ }^{10}$ While most of the reported studies deal with individual nanoantennas, arrays of nanoantennas are of special interest. If the nanoantennas are arranged into a periodic array whose period is commensurate with the LSPR's wavelength, diffractive coupling between the antennas can be observed. ${ }^{11}$ In this case, the existence of the so-called Rayleigh anomalies, corresponding to diffraction of light in the plane of the array, ${ }^{12}$ allows for long distance communication between the nanoantennas. As a consequence, the localized and spectrally broad plasmon resonance of each nanoantenna can interfere with in-plane scattered light in a narrow spectral range near the position of the Rayleigh anomaly, leading to a Fano lineshape. ${ }^{13-20}$ This resulting mode is known as a surface lattice resonance (SLR), or lattice plasmon mode. SLRs are spectrally narrower than LSPRs, which means that they exhibit lower inherent losses.

To understand the interest of SLRs for fluorescence enhancement, let us consider the canonical model of a quantum emitter inside a cavity. In this case, the Purcell factor, the physical quantity driving the acceleration of the total decay rate of the emitter, writes: ${ }^{3,21}$

$$
F_{P}=\frac{3}{4 \pi^{2}} \frac{Q}{V}\left(\frac{\lambda}{2 n}\right)^{3}
$$

where $V$ is the effective volume of the cavity mode, ${ }^{22,23}$ and $Q$ the quality factor of the cavity resonance, defined as $Q=\lambda / \Delta \lambda$. Eq. 1 indicates that high $Q$-factor plasmonic reso- 
nances should lead to high Purcell factors and are thus extremely appealing for fluorescence enhancement. The coupling of fluorescent emitters to arrays of nanostructures has been widely studied, ${ }^{24-27}$ including arrays sustaining SLRs. ${ }^{28-33}$ However, those works focused on the case of ensembles of emitters. Studying the emission from single emitters is of critical importance to design efficient single-photon sources based on SLRs. ${ }^{4}$

Herein, we study how a periodic array of plasmonic resonators sustaining high quality factor SLRs affects the fluorescence enhancement of a single emitter. Two geometries of metallic nanoantennas are considered: (i) the simplest case of single nanocylinders, and (ii) dimers of nanoparticles, as this system has been reported to yield extremely large fluorescence enhancement factors. ${ }^{7}$ Using numerical calculations based on the finite-difference time-domain (FDTD) method, we show that SLRs allow to reach significantly higher values for the excitation enhancement (due to higher local field intensity). At the same time, we find that the emitter's emission rate (as modeled by an electric dipole) reaches a value comparable to what is obtained with an isolated nanoantenna. Altogether, we demonstrate that SLRs brought a significant advantage to increase the fluorescence brightness of single emitters in comparison to isolated nanoantennas.

\section{Results}

We consider a square array of gold nanoantennas. The period of the array is $P$, the height of the nanoantennas is $h$. We consider two different nanoantenna geometries. The first geometry, depicted in Figure 1a, is the nanodisk antenna, corresponding to the canonical case of an emitter coupled to a single gold nanodisk, whose diameter is denoted $d$. The emitter is located on the side of the nanocylinder, $10 \mathrm{~nm}$ from the metal surface, at mid-height, and is perpendicular to the metal surface. The emitter-surface distance and the emitter's orientation have been chosen because they correspond to a very favorable case for emission enhancement. ${ }^{34,35}$ The second geometry (Figure 2a) consists of a dimer of nanodisks, also 
known as a gap antenna: two gold nanodisks (with the same diameter $d$ ) are separated by a 20-nm-gap. This geometry is known to yield an extremely large field enhancement inside the gap between the two nanodisks. In this configuration the emitter is located in the center of the gap, at mid-height, parallel to the dimer axis. For the sake of simplicity, for both cases the array is supposed to be in a homogeneous dielectric environment with a refractive index $n=1.5$. It has also been demonstrated that a homogeneous environment is the most favorable geometry to obtain sharp lattice modes. ${ }^{20}$ All computations were performed using a commercial finite difference time-domain (FDTD) method (see Methods for details).

To evidence the presence of surface lattice resonances, we start by computing the extinction spectrum of both nanoantenna arrays. Normal incidence extinction spectra have been computed for an infinite array of gold nanodisks with diameter $d=100 \mathrm{~nm}$ and height $h=50 \mathrm{~nm}$, for periods ranging from 300 to $600 \mathrm{~nm}$. The resulting dispersion curve is shown in Figure 1b. On Figure 1b are also plotted as solid white lines the positions of the Rayleigh anomalies, obtained by the following expression:

$$
\lambda_{m, p}=n P \frac{\sqrt{m^{2}+p^{2}\left(1-\sin ^{2} \theta\right)} \pm m \sin \theta}{m^{2}+p^{2}},
$$

where $\theta$ is the angle of incidence, $n$ is the refractive index of the embedding medium, and $m$ and $p$ are integers corresponding to the diffracted order of the anomaly. ${ }^{20}$ For shorter periods, the dispersion curve evidences a broad resonance around $\lambda=700 \mathrm{~nm}$ corresponding to the (dipolar) LSPR of the nanodisks. When the period increases, the resonance shifts toward the position of the Rayleigh anomaly and significantly sharpens. This effect results from the coupling between the LSPR and the in-plane scattered light ${ }^{20}$ and is best seen in the line cuts presented in Fig. 1c. On the same figure is also plotted the extinction spectrum for an isolated nanodisk, evidencing the remarkable sharpening of the resonance peak obtained with lattice modes. The quality factor of the resonance, as well as its spectral position, can be tuned by changing the period. Fig. 1d shows the value of the quality factor $Q$ as a 

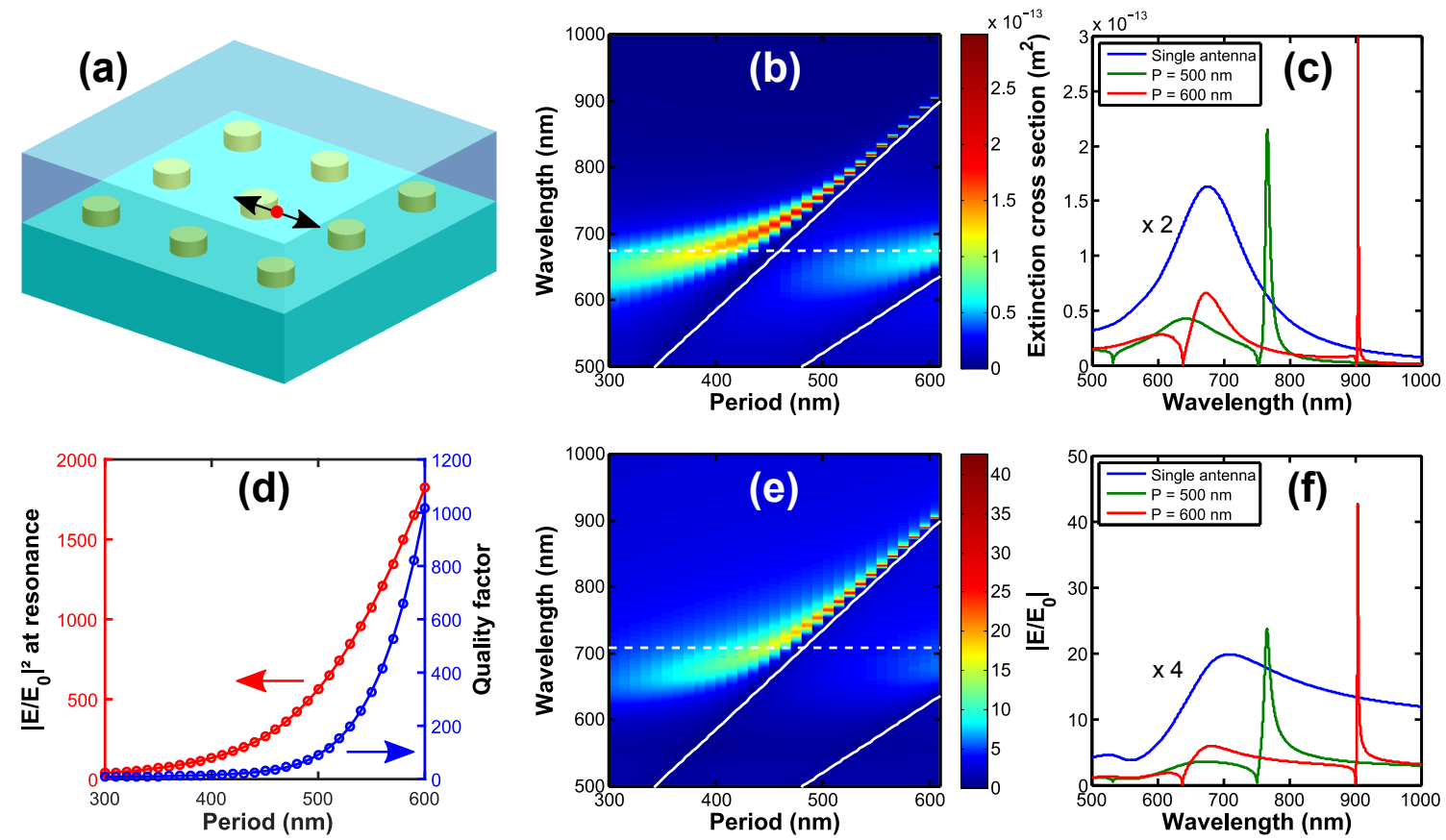

Figure 1: Surface lattice resonances in nanodisks arrays. (a) Schematic of the studied geometry: a square array (with period $P$ ) of gold nanodisks $(d=100 \mathrm{~nm}, h=50 \mathrm{~nm})$ inside a homogeneous medium with refractive index $n=1.5$. (b) Computed extinction cross-section spectra as a function of the period $P$ of the array, under normal incidence. The white solid lines correspond to the position of the Rayleigh anomalies. (c) Extinction spectra for an array with $P=500 \mathrm{~nm}$ (green line), $P=600 \mathrm{~nm}$ (red line), and for an individual antenna (blue line). (d) Quality factor (blue dots) and normalized field intensity $\left|\mathrm{E} / \mathrm{E}_{0}\right|^{2}$ computed at resonance (red dots) of the lattice mode as a function of the period of the array. (e) Normalized electric field modulus $\left|\mathrm{E} / \mathrm{E}_{0}\right|$ as a function of the wavelength and the period, under normal incidence. (f) Normalized field modulus $\left|\mathrm{E} / \mathrm{E}_{0}\right|$ spectra (calculated at the emitter's position) for an array with $P=500 \mathrm{~nm}$ (green line), $P=600 \mathrm{~nm}$ (red line) and for an individual antenna (blue line). For (d-f), the electric field is computed $10 \mathrm{~nm}$ away from the nanoparticle, at mid-height.

function of the period (blue dots). It appears that the quality factor obtained with a SLR can be two orders of magnitude higher than the one of an isolated nanodisk. Similar effects are observed for an array of gap antennas (see Figure 2b-d). However, in comparison with nanodisks, gap antennas yield lower Q-factors as near-field coupling between the particles forming the dimer broadens the resonance. 

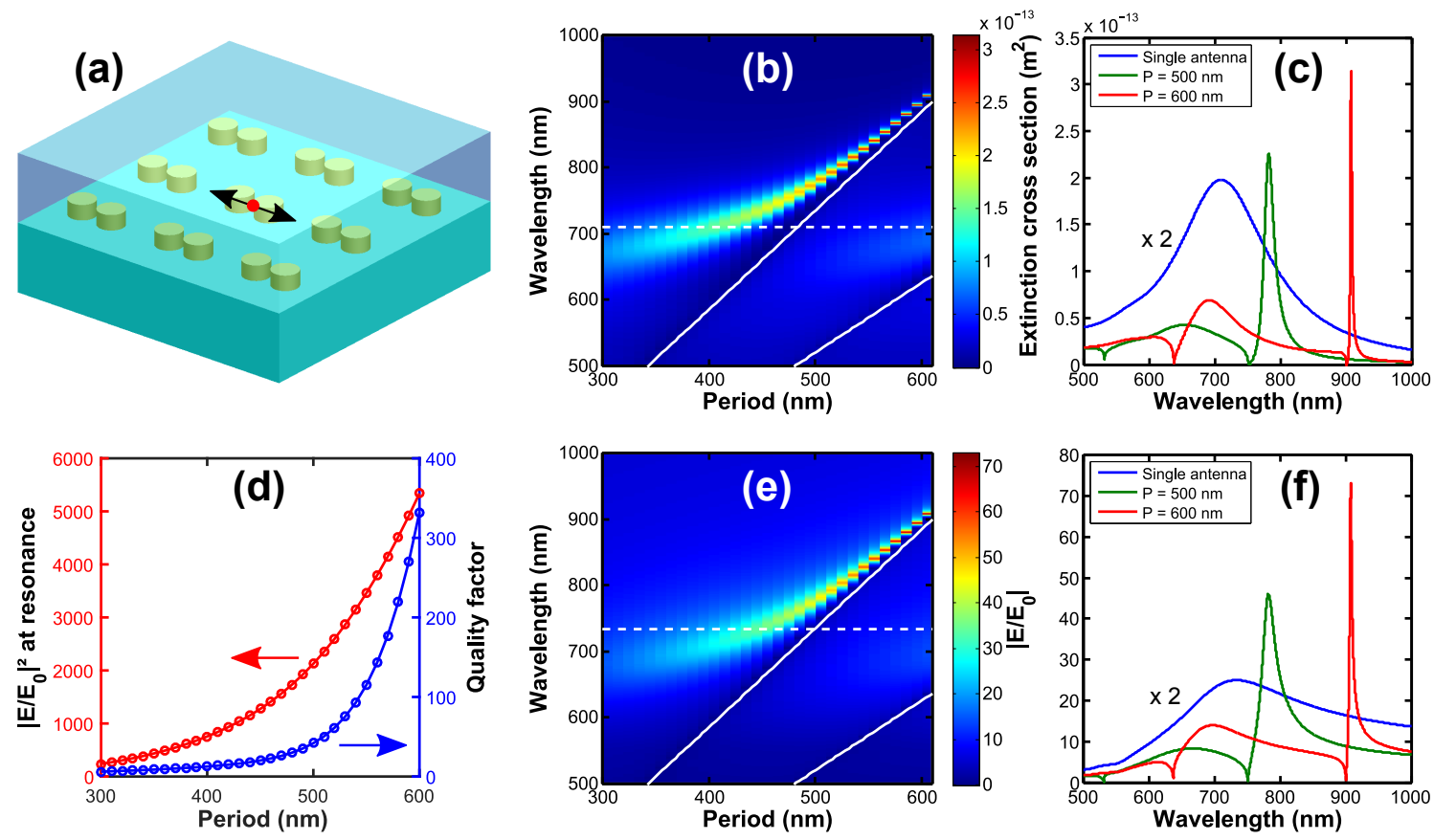

Figure 2: Same as Fig. 1, for arrays made of gap antennas.

\section{Excitation enhancement}

To study the effect of periodicity on the local field intensity (and thus on the fluorescence excitation efficiency), we plotted in Figure 1e the normalized local electric field modulus $\left|\mathrm{E} / \mathrm{E}_{0}\right|$ computed at the emitter's position (red dot in Figure 1a), as functions of both the period of the array and the wavelength. The arrays are illuminated with a plane wave under normal incidence. It appears that the local field enhancement follows a trend very similar to extinction cross-section, as the field is locally enhanced where a resonance is excited. To go further, we plotted in Figure 1f the field enhancement as a function of the wavelength for two different periods and for an isolated nanodisk. Comparing Figures 1c and 1f evidences a direct correlation between the quality factor and the local field intensity in the vicinity of the nanoparticles: the sharper the resonance, the higher the local electric field. This result is confirmed by comparing the blue and red curves in Figure 1d, showing both the quality factor and the normalized field intensity $\left|\mathrm{E} / \mathrm{E}_{0}\right|^{2}$ at resonance (i.e. for the maximum value of the peak in Figure 1f) as a function of the period of the array. The dramatic effect of the periodicity is clearly evidenced. While the maximum electric field intensity enhancement for 
an isolated antenna is $\sim 25$ (see Figure 1f, blue curve), values higher than 1000 are easily reached at resonance for the array. This 40-fold enhancement of the local field intensity is directly linked to the spectral sharpening of the resonance. Similar calculations have been performed for the gap antennas and are reported in Figure 2. In particular, Figure 2d shows that the local field intensity monitored at the center of the gap can reach 5000 times the incident intensity. Again, the obtained local field intensity is orders of magnitude higher than what can be obtained with a single nanoantenna, demonstrating the interest of working with lattice modes rather than localized resonances.

The enhancement of the local field intensity is a direct consequence of the existence of the SLR. Being the result of the hybridation between a localized resonance (the LSP) and a delocalized resonance (the photonic mode close to the Rayleigh anomaly), the SLR allows a better harvesting of the incident plane wave, efficiently concentrating the field inside the nanogap. In other words, near the SLR the array acts as a light concentrator, increasing light intensity in the vicinity of the nanoantennas.

\section{Quantum yield enhancement}

Now we turn our attention to the fluorescence emission enhancement. We model the emission by considering a single emitter (electric dipole) located inside a finite array of nanoantennas. The use of a finite array is due to numerical reasons: infinite arrays being modeled with periodic boundary conditions on the sides of the simulation box, this will lead to the computation of the emission from an infinite array of emitters oscillating in phase. Hence, we compute the emission from a single dipole, coupled to an antenna located at the center of a $25 \times 25$ antenna array. Then, we compute the radiative enhancement factor $f_{r}$, the non-radiative factor $f_{n r}$ and the quantum yield enhancement factor $f_{\eta}=\eta / \eta^{(0)}$ (see Methods). The emitter is assumed to exhibit an initial quantum yield $\eta^{(0)}=1 \%$ (corresponding to the quantum yield inside the homogeneous medium without any antenna). Results are summarized in Fig. 3 for a finite array of gold nanodisk antennas, and in the S.I. (Figure 

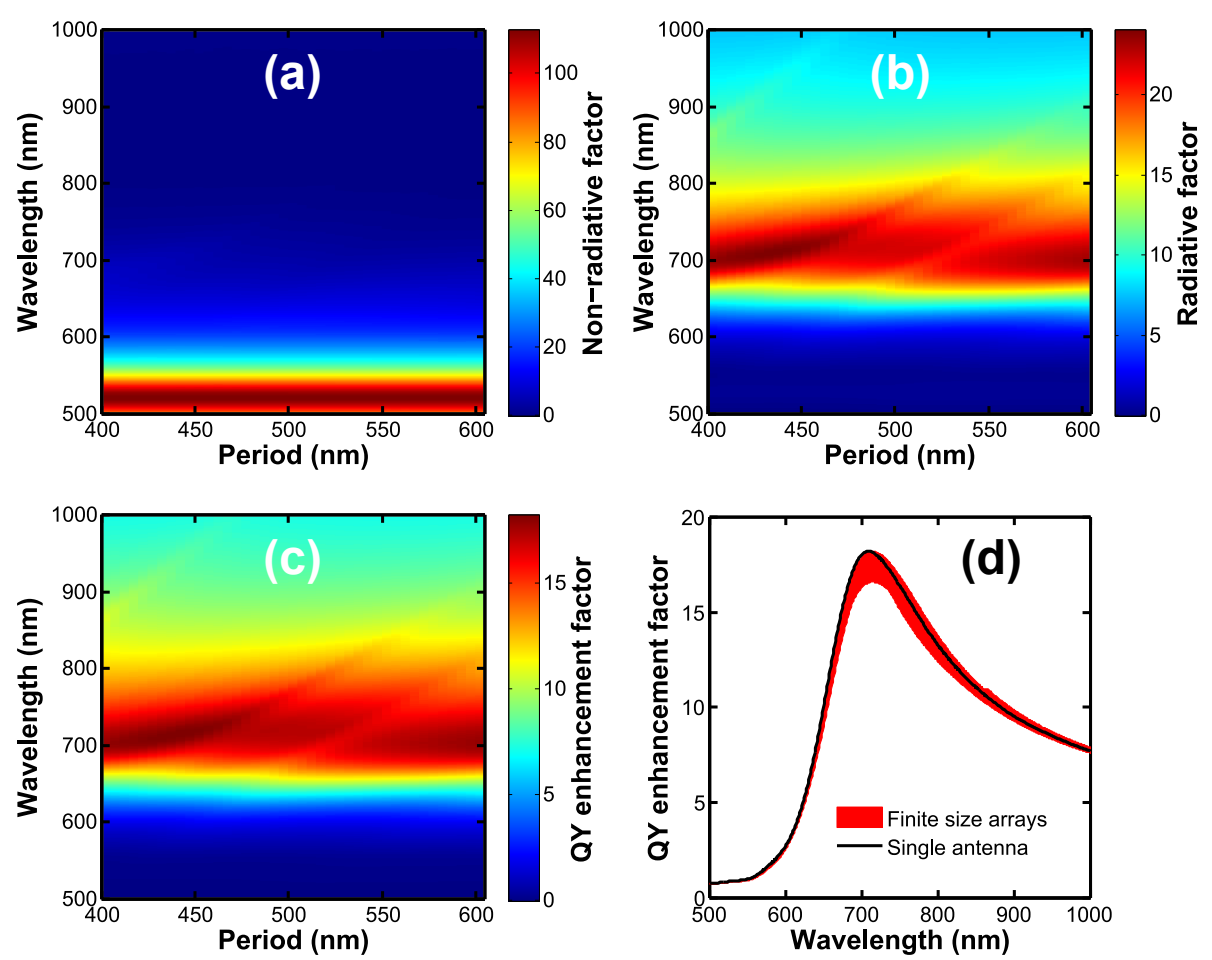

Figure 3: Lattice effect on the emission for gold nanodisks $(d=100 \mathrm{~nm}, h=50 \mathrm{~nm})$. (a) Non-radiative factor $f_{n r}$ as a function the period $P$ and the wavelength $\lambda$. (b) Same, for the radiative factor $f_{r}$. (c) Same, for the quantum yield enhancement factor. (d) Quantum yield enhancement factor spectra, for periods ranging from 400 to $600 \mathrm{~nm}$ (red shaded area) and for a single antenna (black solid curve).

S1) for gap antennas.

Figure 3 a shows the non-radiative factor $f_{n r}$ as a function of the wavelength and the period of the array. It evidences clearly that, whatever the period, the coupling with the array generates non-radiative losses in the spectral range around $\lambda=520 \mathrm{~nm}$. This wavelength corresponds to the onset of interband transitions in gold, showing that non-radiative processes are mostly governed by the nature of the metal and are not affected by the geometry of the array. A closer inspection of Figure 3a evidences a slight increase of the non-radiative factor in the range $550-650 \mathrm{~nm}$, weakly depending on the period, which corresponds to the excitation of higher-order LSPRs (quadrupolar resonance). The radiative rate (Figure 3b) is enhanced in a relatively broad spectral range corresponding to the position of the dipolar LSP resonance. Looking closely at Figure 3b, it appears that the spectrum is slightly modified when the period is changed. However, the amplitude of the variation is limited: 


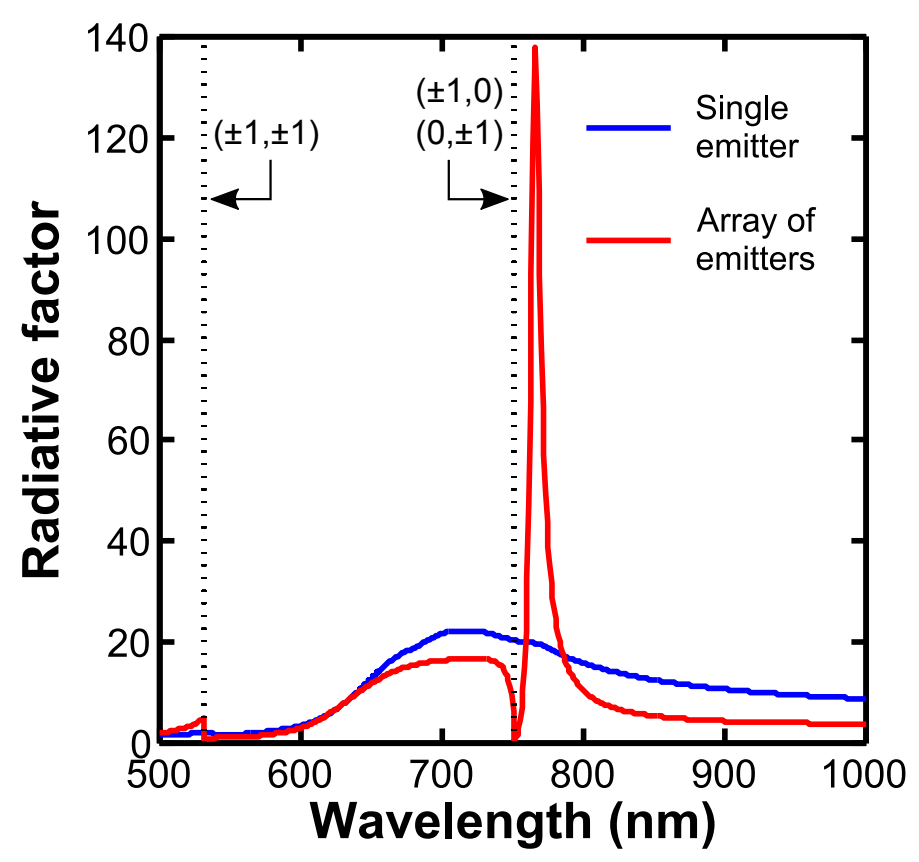

Figure 4: Radiative factor for an array of coherently emitting dipoles coupled to an array of gold nanodisks $(P=500 \mathrm{~nm}, d=100 \mathrm{~nm}, h=50 \mathrm{~nm})$. The initial quantum yield of each emitter is $\eta^{(0)}=1 \%$.

for instance the value of $f_{r}$ oscillates between 25 and 20 at $\lambda=700 \mathrm{~nm}$. The quantum yield enhancement (Figure 3c) exhibits a very similar behavior: changing the period of the array does not imply dramatic effects on the decay rate of a single emitter. The amplitude of variation is further illustrated in the spectra from Figure 3d, showing as a black line the quantum yield enhancement factor for a single emitter coupled to a single nanoantenna, and in red the superimposition of the spectra for arrays with periods from 300 to $600 \mathrm{~nm}$. Figure $3 \mathrm{~d}$ demonstrates that the array does not bring any real beneficial effect with respect to a single nanoantenna - the maximum relative gain in quantum yield is less than $1 \%$. Very similar results are observed for the gap antennas (see S.I.). Figure S1d shows that arrays of gap-antennas do not bring any gain in quantum yield when compared to a single gap antenna.

After what was observed in the previous Section for the excitation enhancement, this result might appear as a surprise. It was shown previously that the field monitored at a given wavelength $\lambda$ in the vicinity of a nanoantenna could be significantly enhanced providing 
those antennas were arranged into an array. Conversely, it seems that an emitter located at the same position, and emitting at the same wavelength $\lambda$ inside the same array, emits light at roughly the same rate it would do near a single antenna. In other words, while SLRs can significantly enhance the excitation rate of a single emitter, they appear to be useless for the enhancement of its emission. However, the array significantly impacts the decay rates in the case of an array of emitters coupled to nanoantennas, all emitting in phase. ${ }^{29,30}$ Such a situation is considered in Figure 4, where we compare the radiative factor of a single emitter (blue line) and of an array of coherent emitters (red line), both coupled to the same array of gold nanodisks. A striking difference is observed between the curves. For the array of emitters, one retrieves the Fano shape, which is characteristic of the extinction spectrum of SLRs (see Figure 1c), associated with high values of the radiative factor $\left(f_{r} \simeq 140\right)$. In contrast, the single emitter exhibits a broad spectrum similar to the extinction spectrum of a single gold nanodisk, with limited values of the radiative factor $\left(f_{r} \simeq 20\right)$.

Generally speaking, the quantum yield enhancement factor critically depends on the value of the intrinsic quantum yield of the emitter. ${ }^{36}$ However, we underline that our results do not depend on our arbitrary choice of $\eta^{(0)}=1 \%$. Computations have been performed for different values of $\eta^{(0)}$ and show no clear influence of the array, even for extremely high or low values of the intrinsic quantum yield (see S.I., Figure S2).

\section{Beaming and radiation pattern}

The modification of the quantum yield is not the only effect that can affect fluorescence emission. Interaction with a nanoantenna can also modify the emitter's radiation pattern, leading to light beaming or redirection. ${ }^{6}$ In the case of arrays of nanoantennas, it has been shown recently that the beaming effect can be reinforced ${ }^{37}$ or give rise to structured emis-

sion. ${ }^{17,38,39}$ Structured emission is well-known in antenna phased arrays and corresponds to higher emission in specific directions where the scattered light from each antenna interferes constructively, due to the phase relation between each scatterer. ${ }^{40}$ Therefore, even if the 

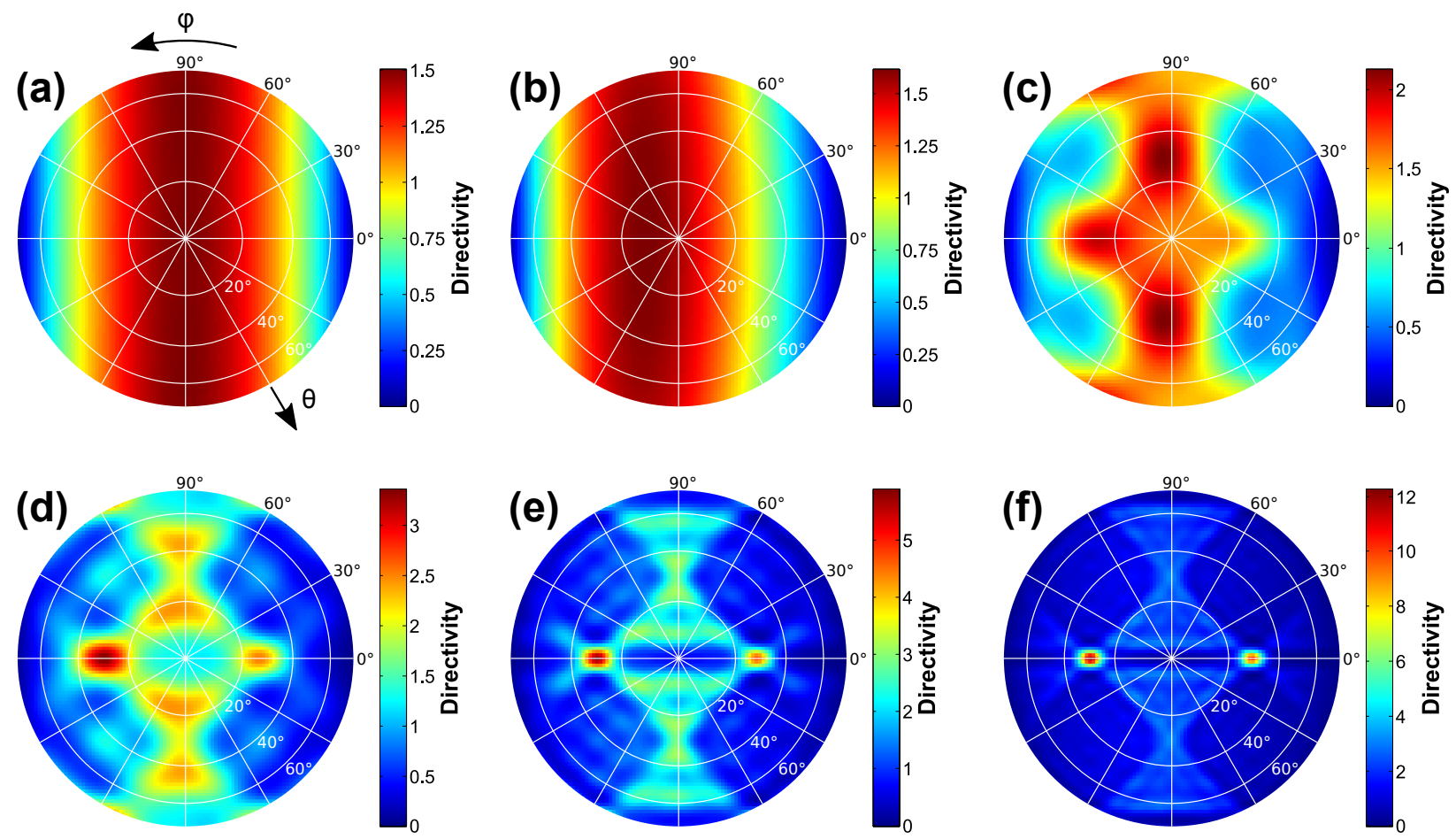

Figure 5: Far-field radiation pattern of finite arrays of nanodisk antennas, for different sizes of the array. The period of the array is $P=500 \mathrm{~nm}$. The diagrams are plotted for the emission wavelength corresponding to the peak value of the emission rate. (a) Emitter only, $\lambda=707 \mathrm{~nm}$. (b) Emitter coupled to a single antenna, $\lambda=707 \mathrm{~nm}$. (c) Emitter in a $3 \times 3$ array, $\lambda=723 \mathrm{~nm}$. (d) Emitter in a $5 \times 5$ array, $\lambda=725 \mathrm{~nm}$. (e) Emitter in a $9 \times 9$ array, $\lambda=717 \mathrm{~nm}$. (f) Emitter in a $15 \times 15$ array, $\lambda=715 \mathrm{~nm}$.

emission rate is not impacted by the lattice mode, the collection efficiency of the emitted light will be improved if beaming effects exist.

To ascertain the existence of beaming, the far-field radiation patterns were calculated for different sizes of the array. We started from the emitting dipole alone, then the emitter coupled to a single antenna, and then coupled to arrays with increasing sizes $(3 \times 3,5 \times 5$, $9 \times 9$ and $15 \times 15$ nanoantennas). We chose to plot the antenna directivity $D(\theta, \varphi)$, which measures the ability of an antenna to direct and concentrate light in a given direction $(\theta, \varphi) .{ }^{41}$ Results are presented in Fig. 5 for arrays of nanodisk antennas with a period $P=500 \mathrm{~nm}$, and an emission wavelength $\lambda=750 \mathrm{~nm}$. It appears that the formation of the array strongly affects the emission pattern, the fluorescence light being gradually beamed into two emission lobes as the size of the array is increased. 

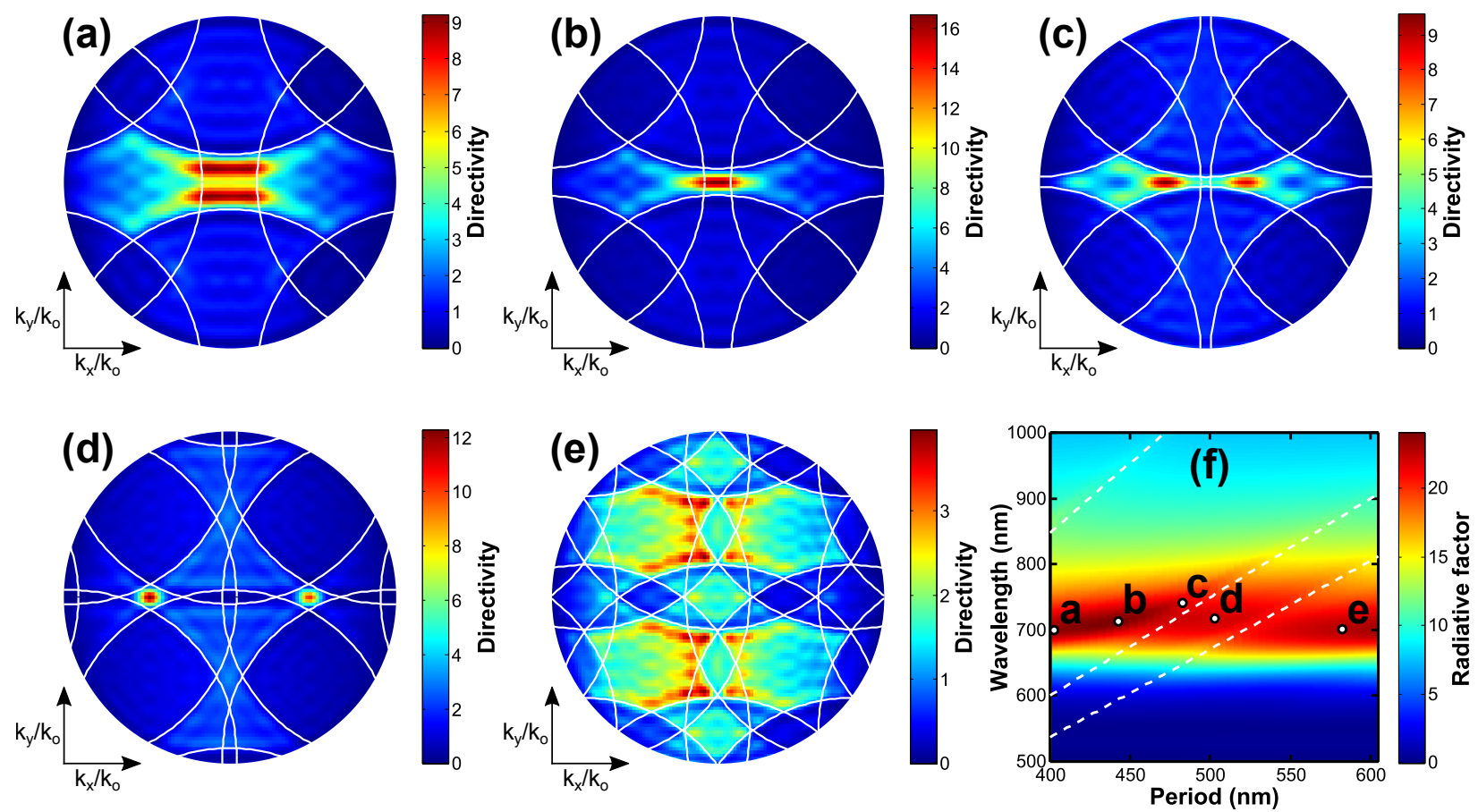

Figure 6: Far-field radiation pattern of a single dipole inside a finite array of nanodisk antennas, for different positions $(P, \lambda)$ in the dispersion diagram. All arrays consist of $15 \times 15$ gold nanodisks. The solid white lines represent the position of the diffraction orders. (a) $P=400 \mathrm{~nm}, \lambda=700 \mathrm{~nm}$. (b) $P=440 \mathrm{~nm}, \lambda=713 \mathrm{~nm}$. (c) $P=480 \mathrm{~nm}, \lambda=741 \mathrm{~nm}$. (d) $P=500 \mathrm{~nm}, \lambda=717 \mathrm{~nm}$. (e) $P=580 \mathrm{~nm}, \lambda=701 \mathrm{~nm}$. (f) Dispersion diagram of the radiative enhancement factor as a function of $P$ and $\lambda$. The positions where the radiation patterns have been calculated are shown as white dots.

More insight can be gained by plotting the far-field radiation patterns for different values of the period $P$ and the emission wavelength $\lambda$, that is to say, at different positions in the dispersion diagram. The results shown in Fig. 6 evidence different emission patterns depending on the coordinates $(P, \lambda)$ on the dispersion diagram. It appears that the emission can be structured either as a single lobe centered around a direction normal to the plane of the sample (for the smallest values of the period), or as two emission lobes (for intermediate values), or as a more complex pattern with multiples lobes (for the largest periods). In fact, three zones can be distinguished on the dispersion diagram, whose boundaries are shown in Fig. 6f by the white dashed lines. The far-field patterns are slightly asymmetric, since the emitter (located on the side of the central nanodisk antenna) breaks the overall symmetry of the square array. In contrast, the far-field radiation patterns for the gap antenna, where the 
(a)

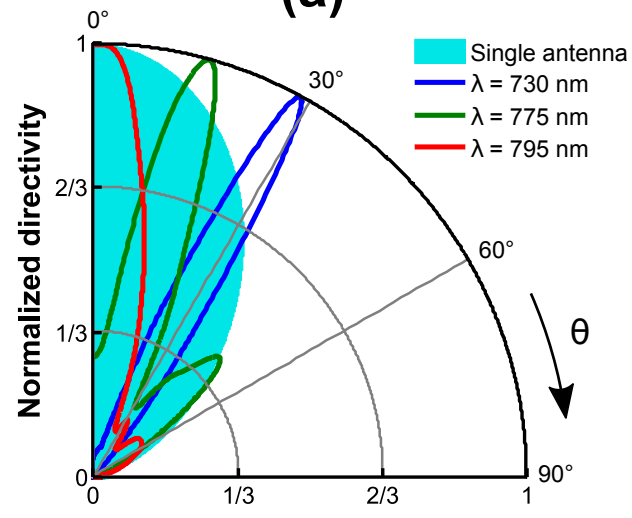

(b)

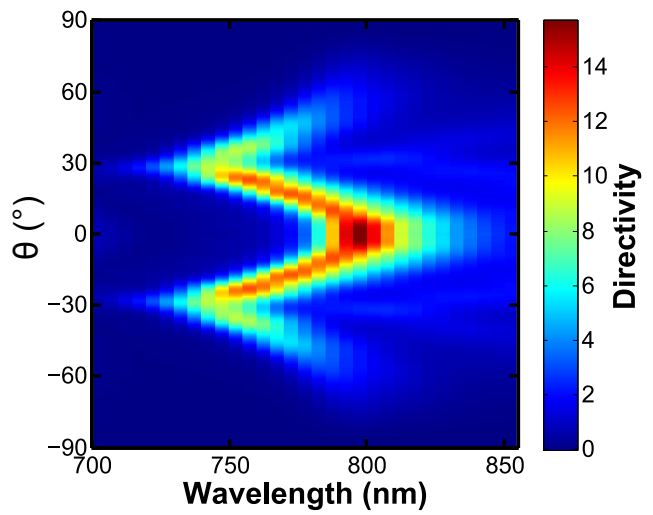

Figure 7: Far-field radiation pattern of a single dipole in a $15 \times 15$ array of gap antennas, for a constant lattice period $P=500 \mathrm{~nm}$. (a) Polar plot showing the normalized directivity $D$ for three values of the wavelength : $\lambda=730 \mathrm{~nm}$ (blue), $\lambda=775 \mathrm{~nm}$ (green) and $\lambda=795 \mathrm{~nm}$ (red). The emission pattern of the dipole inside a single gap antenna is shown for reference as the light blue shaded area. (b) Directivity as a function of wavelength and emission angle.

emitter is located in the center of the gap, are perfectly symmetric (see Figure S3). To understand the formation of the far-field radiation pattern, it is interesting to plot the position of the diffraction orders in the Fourier space, or wavevector diagram. This approach has been previously successfully applied by Rigneault and coworkers to model dipole emission inside a grating for light extraction applications. ${ }^{24}$ The position of the first orders of diffraction (for a plane wave under grazing incidence) has been plotted as white lines in Fig. 6a-e. In the approximation where the periodic structure is only weakly perturbing the dipole radiation (which is obviously not the case here), the far-field radiation would be along the directions defined by those arcs. ${ }^{24}$ In our case, the emission lobes are located near the intersection of several diffraction orders, and the polarization of the emitting dipole has to be taken into account.

The far-field radiation patterns evidence that the angle of emission is controlled by the dispersion diagram. Furthermore, for a given period of the array, the angle of emission depends on the emitting wavelength. This is evidenced in Fig. 7, showing the far-field emission for different wavelengths and a constant period $P=500 \mathrm{~nm}$. For the sake of simplicity, we plot in Fig. 7 the results for a gap antennas, as they yield symmetrical patterns. 
Fig. 7a shows polar plots of the far-field emission (directivity) for different wavelengths and a period $P=500 \mathrm{~nm}$. All patterns have been normalized to unity, and the far-field emission of a dipole inside a single antenna is also shown for reference. In the relatively narrow range of wavelengths explored in Fig. 7a, very different emission angles are observed, from 0 to $30^{\circ}$. Moreover, for each wavelength the emission cone is narrow, meaning that color-dependent photon sorting is possible. ${ }^{42}$ Fig. $7 \mathrm{~b}$ is a color plot of the emission angle as a function of the wavelength, and shows that tuning of the emission angle is possible by changing the wavelength. A transition from a two-lobe emission pattern to a pattern exhibiting one main lobe is observed around $780 \mathrm{~nm}$.

\section{Discussion}

In this Section we assess the interest of using SLRs in the context of single emitter fluorescence enhancement, by comparing our results with what was previously reported in the literature - keeping in mind that the enhancement factor is emitter-dependent. ${ }^{36}$ Assuming a two-level quantum emitter excited well below its saturation intensity, the total fluorescence (i.e., emitter brightness) enhancement factor $f_{\text {low }}$ can be written as:

$$
f_{\text {low }}=f_{\text {exc }} \cdot f_{\eta} \cdot f_{\text {col }}
$$

where $f_{\text {exc }}=\left|\mathrm{E} / \mathrm{E}_{0}\right|^{2}$ is the enhancement of the excitation, $f_{\eta}=\eta / \eta^{(0)}$ is the enhancement of the quantum yield, and $f_{c o l}$ is the enhancement of the collection efficiency. ${ }^{43}$ In order to clearly evidence the advantage given by surface lattice resonances, we summarized in Table 1 the various fluorescence enhancement factors for an isolated nanoantenna and for an array of nanoantennas. The excitation (resp. emission) wavelength has been chosen as the wavelength yielding to the highest value of the excitation (resp. emission) enhancement factor. The collection enhancement factor has been computed at the same wavelength than $f_{\eta}$. The resulting wavelengths are given in Table 1 and corresponds to realistic values for a 
Table 1: Comparison of the fluorescence enhancements for isolated antennas and arrays of antennas, for two antenna geometries (nanodisks and gap antennas). The period of the array is $P=440 \mathrm{~nm}$. The excitation enhancement factor is computed at $\lambda_{\text {exc }}$, while all the emission-related enhancement factors are computed at $\lambda_{e m i}$. The initial quantum yield is $\eta^{(0)}=1 \%$.

\begin{tabular}{cc|cc|ccc|c} 
& & $f_{\text {exc }}$ & $\lambda_{\text {exc }}$ & $f_{\eta}$ & $f_{\text {col }}$ & $\lambda_{\text {emi }}$ & $f_{\text {low }}$ \\
\hline \multirow{2}{*}{ Nanodisks } & Single antenna & $\mathbf{4 . 9 7}$ & $709 \mathrm{~nm}$ & $\mathbf{1 8 . 2}$ & $\mathbf{1 . 0 3}$ & $709 \mathrm{~nm}$ & $\mathbf{9 3}$ \\
& $15 \times 15$ array & $\mathbf{2 3 0}$ & $707 \mathrm{~nm}$ & $\mathbf{1 8 . 2}$ & $\mathbf{3 . 3}$ & $715 \mathrm{~nm}$ & $\mathbf{1 3 8 0 3}$ \\
\hline \multirow{2}{*}{ Gap antennas } & Single antenna & $\mathbf{1 5 6}$ & $734 \mathrm{~nm}$ & $\mathbf{5 4 . 7}$ & $\mathbf{1 . 0 6}$ & $744 \mathrm{~nm}$ & $\mathbf{9 0 7 5}$ \\
& $15 \times 15$ array & $\mathbf{1 1 4 9}$ & $734 \mathrm{~nm}$ & $\mathbf{5 2 . 8}$ & $\mathbf{3 . 9 7}$ & $748 \mathrm{~nm}$ & $\mathbf{2 4 0 7 7 6}$
\end{tabular}

low Stokes shift emitter. The period of the array $(P=440 \mathrm{~nm})$ has been chosen to maximize the directivity and hence $f_{c o l}$. We also assume that the fluorescence is collected using a low numerical aperture $(N A=0.3)$ microscope objective.

Table 1 unambiguously evidences the advantage brought by the SLR, both for a bare nanodisk antenna or for gap antennas. We emphasize that the studied geometries are easy to make with a standard electron-beam lithography process. The enhancement factors can be compared to the performance metrics given in the recent Perspective article by Koenderink. ${ }^{4}$ Obviously, the huge enhancement factor obtained for an array of gap antennas $\left(f_{\text {low }}=\right.$ $2.4 \times 10^{5}$ ) will be mitigated in a practical experiment, by fabrication imperfections, by the emitter orientation, position and quantum yield, and for the excitation enhancement by the numerical aperture of any excitation lens. It is interesting to compare our results on the single gap antenna with the experiment reported by Kinkhabwala and coworkers, ${ }^{7}$ who measured a total enhancement factor of 1340 using bow-tie gap antennas and low quantum yield $(2.5 \%)$ emitters. Kinkhabwala et al. relied on numerical simulations to infer the excitation and emission enhancements, and obtained $f_{\text {exc }}=180$ and $f_{\eta}=9.3$, with no collection enhancement. Their lower value for the emission enhancement is likely due to the orientation of the emitter and to the higher value of the initial quantum yield. Using an emitter with $\eta^{(0)}=1 \%$ increases the value of $f_{\eta}$ to 21.7 , reasonably close to our value. This comparison shows that the theoretical performances reported in Table 1 
are experimentally achievable, even if the numbers should be understood as upper limits. Moreover, this comparison underlines that there is a lot of room for further improvement of the fluorescence brightness, keeping the same bow-tie antenna geometry arranged into a periodic array. Interestingly, as SLRs also exist in arrays of dielectric optical antennas, ${ }^{44,45}$ arrays of dielectric antennas also offer avenues for the enhancement of fluorescence of single emitters with low-loss materials. ${ }^{46-49}$

\section{Conclusion}

To sum up, we theoretically demonstrated that arrays of plasmonic nanoantennas sustaining surface lattice resonances are highly beneficial for the enhancement of the excitation rate of single emitters. On the one hand, the array does not bring specific advantage on the spontaneous emission rate compared to a single nanoantenna, whatever the geometry of the nanoantenna or the intrinsic quantum yield of the considered emitter. On the other hand, the array both harvests the incident light into nanometric volumes and generates structured emission in the far-field that can increase the collection efficiency of fluorescence. In other words, the array acts as a far- to near-field transducer, that is to say, an antenna. The array of nanoantennas can hence be seen as a "meta-antenna", further increasing the efficiency of its constituting elements. This effect is actually the nano-optical analogue of the enhanced directivity observed in arrays of micro-wave antennas. ${ }^{40}$ Arrays sustaining SLRs can therefore act as on-chip lenses, allowing efficient concentration and extraction of light without requiring the use of high numerical aperture microscope objectives.

\section{Methods}

The whole system is modeled using a commercial FDTD software. ${ }^{50}$ The refractive index of gold has been taken from Ref. ${ }^{51}$ A constant mesh size of $2 \mathrm{~nm}$ was set to define precisely each antenna, while a non-uniform mesh was used outside the antennas. To model an isolated 
particle or a finite array, we used perfectly absorbing layers (PMLs) on the six sides of the computation box. To model an infinite array, we use PMLs on the top and bottom sides, while periodic boundary conditions (PBCs) are applied for the four remaining sides. Let us underline that when dealing with high-Q factor resonances, special attention must be paid to the PML delimiting the simulation box in order to avoid any undesired reflection. Here, we used stretched coordinates PMLs with the 'steep angle' profile as implemented in ${ }^{50}$ and performed extensive convergence tests.

\section{Computation of the quantum yield enhancement}

We use the methods described in Ref. ${ }^{7}$ The emitter's initial quantum yield (taken in the medium with refractive index $n$ ) is:

$$
\eta^{(0)}=\frac{\gamma_{r}^{(0)}}{\gamma_{r}^{(0)}+\gamma_{n r}^{(0)}}
$$

where $\gamma_{r}^{(0)}$ is the emitter's initial radiative emission rate and $\gamma_{n r}^{(0)}$ its non-radiative rate. When the emitter is coupled to the nanoantenna, its apparent quantum yield writes:

$$
\eta=\frac{\gamma_{r}}{\gamma_{r}+\gamma_{n r}}
$$

In Eq. $5, \gamma_{r}$ is the radiative rate of the coupled system and $\gamma_{n r}=\gamma_{n r}^{(0)}+\gamma_{a b s}$ its non-radiative rate. For the latter, $\gamma_{a b s}$ accounts for all the new absorption decay channels that are brought by the nanoantenna. It is then possible to compute the radiative rate enhancement factor $f_{r}$ as:

$$
f_{r}=\frac{\gamma_{r}}{\gamma_{r}^{(0)}}=\frac{P_{r}}{P_{r}^{(0)}}
$$

where $P_{r}$ is the power radiated by the emitter coupled with the nanoantenna and $P_{r}^{(0)}$ is the power radiated by the emitter into vacuum. ${ }^{41}$ The non-radiative rate factor $f_{n r}$ can be 
calculated as:

$$
f_{n r}=\frac{\gamma_{a b s}}{\gamma_{r}^{(0)}}=\frac{P_{a b s}}{P_{r}^{(0)}},
$$

where $P_{a b s}$ is the power absorbed by the antenna. By combining Eq. 6 and 7 into Eq. 4 and 5, one obtains the expression for the quantum yield enhancement factor:

$$
f_{\eta}=\frac{\eta}{\eta^{(0)}}=\frac{f_{r}}{\left(f_{r}+f_{n r}-1\right) \eta^{(0)}+1} .
$$

\section{Computation of the antenna radiation pattern}

In antenna theory, the ability of an antenna to concentrate light in a given direction $(\theta, \varphi)$ is quantified by the directivity $D(\theta, \varphi)$, defined as the ratio between the the far-field intensity scattered in the direction $(\theta, \varphi)$ and a reference isotropic radiator, that is:

$$
D(\theta, \varphi)=\frac{I(\theta, \varphi)}{I_{0}}
$$

To compute the scattered intensity $I(\theta, \varphi)$, we first perform a near-field to far-field projection onto the surface $S$ of a half-sphere exhibiting a 1 meter radius, using a procedure implemented in. ${ }^{50}$ This projection yields the electric field in a given direction on the surface of the sphere, denoted $\mathrm{E}(\theta, \varphi)$. Assuming that in far-field, we are dealing with plane waves, it is possible to write the intensity as:

$$
I(\theta, \varphi)=\frac{n}{2} \sqrt{\frac{\varepsilon_{0}}{\mu_{0}}}|\mathrm{E}(\theta, \varphi)|^{2} .
$$

$I_{0}$ being a reference isotropic radiator, it is easily computed as the total scattered power equally distributed over all possible directions, $I_{0}=\frac{P_{r a d}}{4 \pi}$.

Hence, the directivity corresponds to the angular power density normalized by a reference isotropic radiator. ${ }^{41}$ Directions where $D>1$ corresponds to directions with enhanced scattered intensity. 


\section{Acknowledgement}

This work was partially supported by the HPC Center of Champagne-Ardenne ROMEO.

\section{Supporting Information Available}

The following files are available free of charge.

- SuppInfo: Enhanced fluorescence with dimers of gold nanocylinders (Figure S1). Influence of the intrinsic quantum yield of the emitter on the emission enhancement (Figure S2). Radiation patterns for dimers of gold nanocylinders (Figure S3). Equation describing the orders of diffraction (Eq. S1).

This material is available free of charge via the Internet at http://pubs.acs.org/.

\section{References}

(1) Novotny, L.; Van Hulst, N. Nature Photon. 2011, 5, 83-90.

(2) Giannini, V.; Fernández-Domínguez, A. I.; Heck, S. C.; Maier, S. A. Chem. Rev. 2011, $111,3888-3912$.

(3) Pelton, M. Nature Photon. 2015, 9, 427-435.

(4) Koenderink, A. F. ACS Photon. 2017, 4, 710-722.

(5) Zayats, A. V.; Smolyaninov, I. I.; Maradudin, A. A. Phys. Rep. 2005, 408, 131-314.

(6) Curto, A. G.; Volpe, G.; Taminiau, T. H.; Kreuzer, M. P.; Quidant, R.; van Hulst, N. F. Science 2010, 329, 930-933.

(7) Kinkhabwala, A.; Yu, Z.; Fan, S.; Avlasevich, Y.; Muellen, K.; Moerner, W. E. Nature Photon. 2009, 3, 654-657. 
(8) Punj, D.; Mivelle, M.; Moparthi, S. B.; van Zanten, T. S.; Rigneault, H.; van Hulst, N. F.; García-Parajó, M. F.; Wenger, J. Nature Nanotechnol. 2013, 8, 512-516.

(9) Hoang, T. B.; Akselrod, G. M.; Argyropoulos, C.; Huang, J.; Smith, D. R.; Mikkelsen, M. H. Nat. Commun. 2015, 6, 6:8788.

(10) Bidault, S.; Devilez, A.; Maillard, V.; Lermusiaux, L.; Guigner, J.-M.; Bonod, N.; Wenger, J. ACS Nano 2016, 10, 4806-4815.

(11) Ross, M. B.; Mirkin, C. A.; Schatz, G. C. J. Phys. Chem. C 2016, 120, 816-830.

(12) Bonod, N.; Neauport, J. Adv. Opt. Photonics 2016, 8, 156-199.

(13) Zou, S.; Janel, N.; Schatz, G. C. J. Chem. Phys. 2004, 120, 10871-10875.

(14) Auguie, B.; Barnes, W. L. Phys. Rev. Lett. 2008, 101, 143902.

(15) Zhou, W.; Dridi, M.; Suh, J.; Kim, C.; Wasielewski, M.; Schatz, G. C.; Odom, T. W. Nature Nanotechnol. 2013, 8, 506-511.

(16) Väkeväinen, A.; Moerland, R.; Rekola, H.; Eskelinen, A.-P.; Martikainen, J.-P.; Kim, D.-H.; Törmä, P. Nano Lett. 2013, 14, 1721-1727.

(17) Lozano, G.; Grzela, G.; Verschuuren, M. A.; Ramezani, M.; Gómez Rivas, J. Nanoscale 2014, 6, 9223-9.

(18) Yang, A.; Li, Z.; Knudson, M. P.; Hryn, A. J.; Wang, W.; Aydin, K.; Odom, T. W. ACS Nano 2015, 9, 11582-11588.

(19) Yang, A.; Hoang, T. B.; Dridi, M.; Deeb, C.; Mikkelsen, M. H.; Schatz, G. C.; Odom, T. W. Nat. Commun. 2015, 6:6939, doi:10.1038/ncomms7939.

(20) Khlopin, D.; Laux, F.; Wardley, W. P.; Martin, J.; Wurtz, G. A.; Plain, J.; Zayats, A. V.; Dickson, W.; Gérard, D. J. Opt. Soc. Am. B 2017, 34, 691-700. 
(21) Colas des Francs, G.; Barthes, J.; Bouhelier, A.; Weeber, J. C.; Dereux, A.; Cuche, A.; Girard, C. J .Opt. 2016, 18, 1-23.

(22) Koenderink, A. F. Opt. Lett. 2010, 35, 4208-4210.

(23) Sauvan, C.; Hugonin, J. P.; Maksymov, I. S.; Lalanne, P. Phys. Rev. Lett. 2013, 110, 237401.

(24) Rigneault, H.; Lemarchand, F.; Sentenac, A. J. Opt. Soc. Am. A 2000, 17, 1048-1058.

(25) Andrew, P.; Barnes, W. L. Phys. Rev. B 2001, 64, 125405.

(26) Sun, G.; Khurgin, J. B.; Soref, R. A. J. Opt. Soc. Am. B 2008, 25, 1748-1755.

(27) Arbel, D.; Berkovitch, N.; Nevet, A.; Peer, A.; Cohen, S.; Ritter, D.; Orenstein, M. Opt. Express 2011, 19, 9807-9813.

(28) Vecchi, G.; Giannini, V.; Gómez Rivas, J. Phys. Rev. Lett. 2009, 102, 146807.

(29) Pellegrini, G.; Mattei, G.; Mazzoldi, P. J. Phys. Chem. C 2011, 115, 24662-24665.

(30) Teperik, T. V.; Degiron, A. Phys. Rev. Lett. 2012, 108, 147401.

(31) Rodriguez, S. R. K.; Lozano, G.; Verschuuren, M. A.; Gomes, R.; Lambert, K.; De Geyter, B.; Hassinen, A.; Van Thourhout, D.; Hens, Z.; Gómez Rivas, J. Appl. Phys. Lett. 2012, 100, 111103.

(32) Zakharko, Y.; Graf, A.; Schießl, S. P.; Hähnlein, B.; Pezoldt, J.; Gather, M. C.; Zaumseil, J. Nano Lett. 2016, 16, 3278-3284.

(33) Zakharko, Y.; Held, M.; Graf, A.; Rödlmeier, T.; Eckstein, R.; Hernandez-Sosa, G.; Hähnlein, B.; Pezoldt, J.; Zaumseil, J. ACS Photon. 2016, 3, 2225-2230.

(34) Anger, P.; Bharadwaj, P.; Novotny, L. Phys. Rev. Lett. 2006, 96, 113002. 
(35) Kühn, S.; Hakanson, U.; Rogobete, L.; Sandoghdar, V. Phys. Rev. Lett. 2006, 97, 017402 .

(36) Wenger, J. Int. J. Opt. 2012, 2012, 828121.

(37) Langguth, L.; Punj, D.; Wenger, J.; Koenderink, A. F. ACS Nano 2013, 7, 8840-8.

(38) Lozano, G.; Louwers, D. J.; Rodríguez, S. R. K.; Murai, S.; Jansen, O. T. A.; Verschuuren, M. A.; Gómez Rivas, J. Light: Science \&3 Applications 2013, 2, e66.

(39) Guo, K.; Lozano, G.; Verschuuren, M. A.; Gómez Rivas, J. J. Appl. Phys. 2015, 118, 073103.

(40) Balanis, C. A. Antenna theory, 2nd ed.; John Wiley \& Sons, 1997.

(41) Novotny, L.; Hecht, B. Principles of Nano-Optics, 2nd ed.; Cambridge University Press, 2012.

(42) Aouani, H.; Mahboub, O.; Devaux, E.; Rigneault, H.; Ebbesen, T. W.; Wenger, J. Nano Lett. 2011, 11, 2400-2406.

(43) Wenger, J.; Gérard, D.; Bonod, N.; Popov, E.; Rigneault, H.; Dintinger, J.; Mahboub, O.; Ebbesen, T. Opt. Express 2008, 16, 3008.

(44) Evlyukhin, A. B.; Reinhardt, C.; Seidel, A.; Luk'yanchuk, B. S.; Chichkov, B. N. Phys. Rev. B 2010, 82, 045404.

(45) Kataja, M.; Hakala, T. K.; Julku, A.; Huttunen, M. J.; van Dijken, S.; Törmä, P. Nat Comms 2015, 6, doi:10.1038/ncomms8072.

(46) Rolly, B.; Bebey, B.; Bidault, S.; Stout, B.; Bonod, N. Phys. Rev. B 2012, 85, 245432.

(47) Albella, P.; Poyli, M. A.; Schmidt, M. K.; Maier, S. A.; Moreno, F.; Sáenz, J. J.; Aizpurua, J. J. Phys. Chem. C 2013, 117, 13573-13584. 
(48) Staude, I.; Khardikov, V. V.; Fofang, N. T.; Liu, S.; Decker, M.; Neshev, D. N.; Luk, T. S.; Brener, I.; Kivshar, Y. S. ACS Photon. 2015, 2, 172-177.

(49) Regmi, R.; Berthelot, J.; Winkler, P. M.; Mivelle, M.; Proust, J.; Bedu, F.; Ozerov, I.; Begou, T.; Lumeau, J.; Rigneault, H.; García-Parajó, M. F.; Bidault, S.; Wenger, J.; Bonod, N. Nano Lett. 2016, 16, 5143-5151.

(50) FDTD Solutions. Lumerical Solutions.

(51) Johnson, P. B.; Christy, R. W. Phys. Rev. B 1972, 6, 4370-4379. 
Graphical TOC Entry

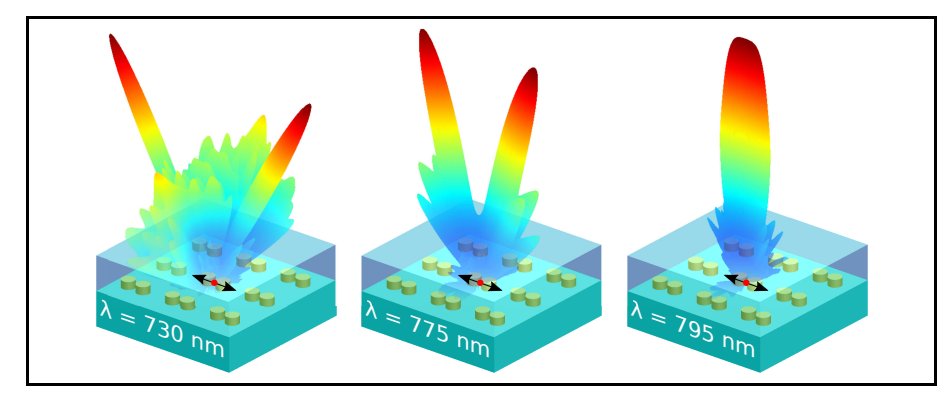

